\title{
Robotic Central Pancreatectomy with Roux-en-Y Pancreaticojejunostomy
}

\author{
Tess M.E. van Ramshorst ${ }^{1}$, Maurice J.W. Zwart ${ }^{1}$, Rogier P. Voermans ${ }^{2}$, Sebastiaan Festen ${ }^{3}$, Freek Daams ${ }^{4}$, Olivier R. \\ Busch $^{1}$, Matthijs W.N. Oomen ${ }^{5}$, Marc G. Besselink ${ }^{1}$ \\ ${ }^{1}$ Department of Surgery, Amsterdam UMC, University of Amsterdam, Cancer Center Amsterdam ${ }^{2}$ Department of Gastroenterology, Amsterdam UMC, \\ University of Amsterdam, Amsterdam Gastroenterology Endocrinology Metabolism ${ }^{3}$ Department of Surgery, OLVG ${ }^{4}$ Department of Surgery, Amsterdam \\ UMC, Vrije Universiteit ${ }^{5}$ Department of Pediatric Surgery, Amsterdam UMC, Emma Children's Hospital
}

\section{Corresponding Author}

Marc G. Besselink

m.g.besselink@amsterdamumc.nl

\section{Citation}

van Ramshorst, T.M., Zwart, M.J., Voermans, R.P., Festen, S.,

Daams, F., Busch, O.R., Oomen, M.W., Besselink, M.G., , Robotic Central

Pancreatectomy with Roux-en-Y

Pancreaticojejunostomy. J. Vis. Exp.

(177), e62862, doi:10.3791/62862

(2021).

\section{Date Published}

November 20, 2021

DOI

$10.3791 / 62862$

\section{URL}

jove.com/video/62862

\section{Abstract}

Central pancreatectomy is a parenchyma-sparing alternative to distal pancreatectomy in patients with a benign or low-grade malignant tumor in the body of the pancreas. The aim of central pancreatectomy is to prevent postoperative life-long endocrine and exocrine insufficiency. The downside of central pancreatectomy is the high rate of postoperative pancreatic fistula, which is the main reason that many surgeons do not routinely use central pancreatectomy in eligible patients. Most studies report open or laparoscopic central pancreatectomy with a pancreatico-gastrostomy anastomosis in adults. This is the first description of a standardized approach to robotic central pancreatectomy with Roux-en-Y pancreaticojejunostomy reconstruction in an adolescent (16-year-old boy) with a pseudopapillary tumor in the body of the pancreas. The operation time was $248 \mathrm{~min}$ with $20 \mathrm{~mL}$ of blood loss. The postoperative course was uneventful except for the short-term medical treatment for a grade B pancreatic fistula. Robotic central pancreatectomy can be safely applied in selected patients in experienced centers.

\section{Introduction}

Central pancreatectomy has been described as a parenchyma-sparing alternative surgical procedure to distal pancreatectomy for benign or low-grade malignant lesions located in the body of the pancreas ${ }^{1,2}$. Central pancreatectomy will prevent life-long pancreatic endocrine and exocrine dysfunction in most patients. Still, most surgeons chose to perform a distal pancreatectomy over a central pancreatectomy because of the increased risk of postoperative morbidity, mainly due to postoperative pancreatic fistula (POPF) after central pancreatectomy ${ }^{3,4,5}$. 
In fact, central pancreatectomy combines the POPF risk of a distal pancreatectomy and a pancreato-enteric anastomosis.

Most studies on central pancreatectomy advise to perform a pancreatico-gastrostomy anastomosis because of the presumed lower risk of $\mathrm{POPF}^{6,7}$. However, current randomized trials are inconclusive regarding the risk for POPF incidence for pancreatico-gastrostomy and pancreaticojejunostomy $8,9,10$. Furthermore, pancreaticogastrostomy has been suggested to impair long-term pancreatic exocrine function due to gastric mucosal overgrowth $^{11}$. In addition, the minority of surgeons are experienced with pancreatico-gastrostomy in daily clinical practice, which will also reduce enthusiasm for this solution.

Robotic pancreatoduodenectomy, supported by the work of the Pittsburgh University Medical Center team and largescale training programs such as the Dutch LAELAPS-3 and the European LEARNBOT programs, has now become an accepted approach in selected patients in many centers across the world ${ }^{12,13,14}$. For surgeons experienced with robotic pancreatoduodenectomy, including the highly standardized robotic pancreatico-jejunal anastomosis, a robotic central pancreatectomy is an attractive alternative to open or laparoscopic central pancreatectomy or laparoscopic distal pancreatectomy.

In this article, we describe a case of a 16-year-old adolescent patient with a pancreatic lesion suspected for solid pseudopapillary tumor who underwent robotic central pancreatectomy with Roux-en-Y pancreaticojejunostomy reconstruction. Our aim is to demonstrate the technical approach and clinical safety of robotic-assisted central pancreatectomy and its surgical outcomes when performed in high-volume centers by experienced surgeons.

\section{Protocol}

The present protocol follows the ethics guidelines of the Amsterdam UMC. Informed consent was obtained from the patient for this article and the video.

\section{Preoperative management}

1. Perform preoperative imaging to assess the extent of the pancreatic lesion and for indications of vascular involvement.

2. Evaluate the eligibility for the surgical approach and relative contraindications.

\section{Operative setting}

1. Place the patient in French position with the right arm lowered on an arm board alongside the patient and the left arm in a $90^{\circ}$ abduction. Tilt the table $20-30^{\circ}$ to the left and in $20-30^{\circ}$ reverse Trendelenburg position.

2. Perform the required safety check procedures and create a sterile exposition with chlorohexidine.

3. Create a pneumoperitoneum by inserting a Veress needle at Palmer's point, then insufflating to $10-12 \mathrm{~mm}$ $\mathrm{CO}_{2}$.

4. After insufflation, mark the positions of the trocars and place them as shown in Figure 1 and Figure 2 (4 robot trocars, 2 (12 $\mathrm{mm}$ ) laparoscopic tableside surgeon trocars, 1 (5 $\mathrm{mm})$ trocar for snake liver retractor).

5. After insufflation, place a $12 \mathrm{~mm}$ tableside surgeon trocar (preferably, one with a blunt clear dome at the distal end, which encloses a crescent-shaped knife blade) to the right of the umbilicus. 
6. After inspection and removal of the Veress needle, place the four $8 \mathrm{~mm}$ robotic trocars $11-12 \mathrm{~cm}$ from the pancreatic body (i.e., target area). Place the trocar at the patient's right side for robot arm 1, more cranial than the others as it is used for contraction.

7. Place a second $12 \mathrm{~mm}$ table-side surgeon trocar. NOTE: Make sure the distance between all the trocars is approximately $7 \mathrm{~cm}$.

8. Finally, place a $5 \mathrm{~mm}$ trocar in the left upper quadrant to hold the laparoscopic stomach/liver retractor.

9. Install the robot at the right side of the patient and dock the robot arms to the robotic trocars.

NOTE: The final operative setting is shown in Figure 3. Robotic arm 1 holds a cadiere forceps, arm 2 a bipolar forceps, arm 3 the camera and arm 4 a cautery hook. The tableside surgeon is positioned between the patient's legs.

\section{Pancreatic identification and mobilization}

1. Open the lesser sac by transecting the gastrocolic ligament approximately $2 \mathrm{~cm}$ distal from the gastroepiploic vessels with the sealing device, controlled by the tableside surgeon.

2. After this, install the liver retractor ( $5 \mathrm{~mm}$ trocar) in the left upper quadrant just subcostally to retract the stomach and the left side of the liver.

3. Perform an intraoperative ultrasound using a robotic ultrasound probe to identify and locate the tumor and mark the future resection margins with the robotic cautery hook.

4. Mobilize the pancreas first caudally and then cranially using the robotic cautery hook on arm 4 and the laparoscopic sealing device.
5. First, place a $1 / 3$ length vessel loop at the left side of the tumor between the pancreas and the splenic vein.

6. Then, further mobilize the pancreas at the proximal side.

7. Place another $1 / 3$ length vessel loop between the pancreas and the portomesenteric vein.

NOTE: Fix the vessel loops using clips.

8. Use the robot arm 1 to lift the vessel loops cranially to create a clear window and to further complete the mobilization of the pancreatic body between the pancreas and the portomesenteric vein.

\section{Pancreatic transection}

1. First, transect the pancreas at the right side of the tumor, typically ventral from the superior mesenteric vein with an endo-stapler with a vascular cartridge.

NOTE: Closing of the stapler using the grade compression approach as described by Asbun $^{15}$, may take up to $4-5 \mathrm{~min}$.

2. Then take care of venous mobilization.

3. After venous mobilization, transect the pancreas at the left side of the tumor using robotic scissors with diathermia. The pancreatic duct is cut without diathermia, i.e., cold cut at the site of the pancreatic duct.

4. Insert a 4.8 French stent to identify the pancreatic duct after the resection

\section{Window in the mesocolon}

1. Create a window in the avascular part of the transverse mesocolon at the (patient's) left side of the colonic vessels using the cautery hook with the mesocolon in the caudal position. 
NOTE: Usually, this location can be identified by locating the proximal jejunum, which is visible through the mesocolon.

2. Hereafter, place and hold the mesocolon in the cranial position held with arm 1. Identify the window by lifting the transverse colon cranially. Identify the ligament of Treitz.

\section{Creating Roux-limb}

1. Create a small opening in the proximal jejunum approximately $20 \mathrm{~cm}$ distally from the ligament of Treitz to divide the proximal jejunum with an endo-stapler with a vascular cartridge.

2. Divide the mesentery at the jejunal transection site using the sealing device.

\section{Positioning Roux-limb}

1. While lifting the transverse colon cranially using robotic arm 1, pass the Roux limb cranially through the window in the mesocolon.

2. Place the transverse colon back in the caudal position.

3. Fixate the end of the Roux limb together with the end of a $50 \mathrm{~cm}$ suture using robot arm 1 . Use robot arms 2 and 4 to measure a $50 \mathrm{~cm}$ distance to the future $S-S$ jejunojejunostomy.

4. Mark this location with a metal clip on the mesentery and lift the transverse colon cranially again.

\section{Jejuno-jejunostomy}

1. Place a polyglactin $4-08 \mathrm{~cm}$ stay suture through the small bowel at the location of the metal clip and the transected proximal jejunum to facilitate the anastomosis. Use arm 1 to retract this stay suture cranially to a location ventrally of the colon to facilitate stapling.
2. Create two small enterotomies using robotic scissors with diathermia.

3. Then, make a side-to-side jejuno-jejunostomy using an endo-stapler with a vascular cartridge.

4. Close the remaining opening of the anastomosis with a 3-0 $15 \mathrm{~cm}$ barbed suture.

\section{Pancreaticojejunostomy}

1. Position the Roux limb for the end-to-side pancreaticojejunostomy. Place a polyglactin 4-0 $8 \mathrm{~cm}$ suture to close the window in the transverse mesocolon and to fixate the jejunal mesentery.

2. Perform the end-to-side pancreaticojejunostomy using a modified Blumgart technique, which is currently highly standardized by the Pittsburgh group and also used in robotic pancreatoduodenectomy ${ }^{16}$.

3. Mattress

1. Start the anastomosis with three transpancreatic silk 2--0 $20 \mathrm{~cm}$ mattress sutures. Drive the first suture at the cranial side of the pancreas through the pancreas and jejunum from a cranial to a caudal position. Drive the suture backward, similar to the anterior side of the pancreas and retract both ends with robot arm 1.

2. Repeat this step for the remaining two transpancreatic mattress sutures centrally and caudally in the pancreas.

NOTE: Avoid suturing the pancreatic duct by placing a stent in the pancreatic duct.

3. Tie the three sutures without removing the needles and remove the stent afterward.

4. Duct-to-mucosa 
1. Create a small enterotomy in the Roux limb with the robotic scissors in preparation for the future duct-tomucosa sutures.

2. Suture the posterior duct-to-mucosa with 4-5 PDS 5-0 $8 \mathrm{~cm}$ sutures in a clockwise manner.

3. Replace the 4.8 French $6 \mathrm{~cm}$ single loop stent in the pancreatic duct and Roux limb to avoid suturing the pancreatic duct when suturing the anterior duct-tomucosa side.

4. Suture the anterior side with 4-5 PDS 5-0 $8 \mathrm{~cm}$ sutures.

5. Tie the sutures after performing the last suture for optimal exposure.

NOTE: Through the whole duct-to-mucosa procedure arm 2 and arm 4 are equipped with needle drivers and arm 1 is used to retract the sutures cranially and ventrally, thus releasing tension.

5. Buttress

1. Reuse the three transpancreatic silk sutures from the mattress procedure to perform the anterior buttress layer of the pancreaticojejunostomy.

2. For all the three sutures, drive the needle through the jejunum and tie these with the remaining end of its suture to complete the anastomosis.

\section{Drain placement}

1. After finishing the pancreaticojejunostomy, introduce a drain through trocar arm 1 to a position cranial of the pancreaticojejunostomy.

\section{Postoperative management}

1. Remove the nasogastric tube. Start a soft liquid diet and expand to a normal diet in $72 \mathrm{~h}$.

2. Assess serum CRP on postoperative day 3 , day 4 , and day 5. Perform a CT or MRI of the abdomen if CRP on day four is elevated compared to day three.

3. Assess drain amylase on postoperative day 3. Remove the drain when drain amylase levels are less than 3 times the upper limit of normal serum amylase.

\section{Representative Results}

Representative results are shown in Table 1. The operation time was 248 min with a measured blood loss of $20 \mathrm{~mL}$. The postoperative course was uncomplicated. Because of initially high drain amylase the patient received octreotide and antibiotics for 3 days. This treatment was stopped when drain amylase levels normalized on postoperative day 7 , when the drain was also removed. Because of this medical treatment, this was graded as a grade B POPF ${ }^{17}$. A normal diet could be restarted after 4 days. The patient was discharged in good condition on postoperative day 8 . The patient did not develop diabetes or pancreatic exocrine insufficiency during the follow-up.

Pathology assessment revealed a $2.2 \mathrm{~cm}$ solid pseudopapillary tumor (Figure 4) and thus confirming the preoperative diagnosis. Microscopically, an R0 resection was confirmed. One lymph node was excised, in which localization of tumor tissue was not found. 


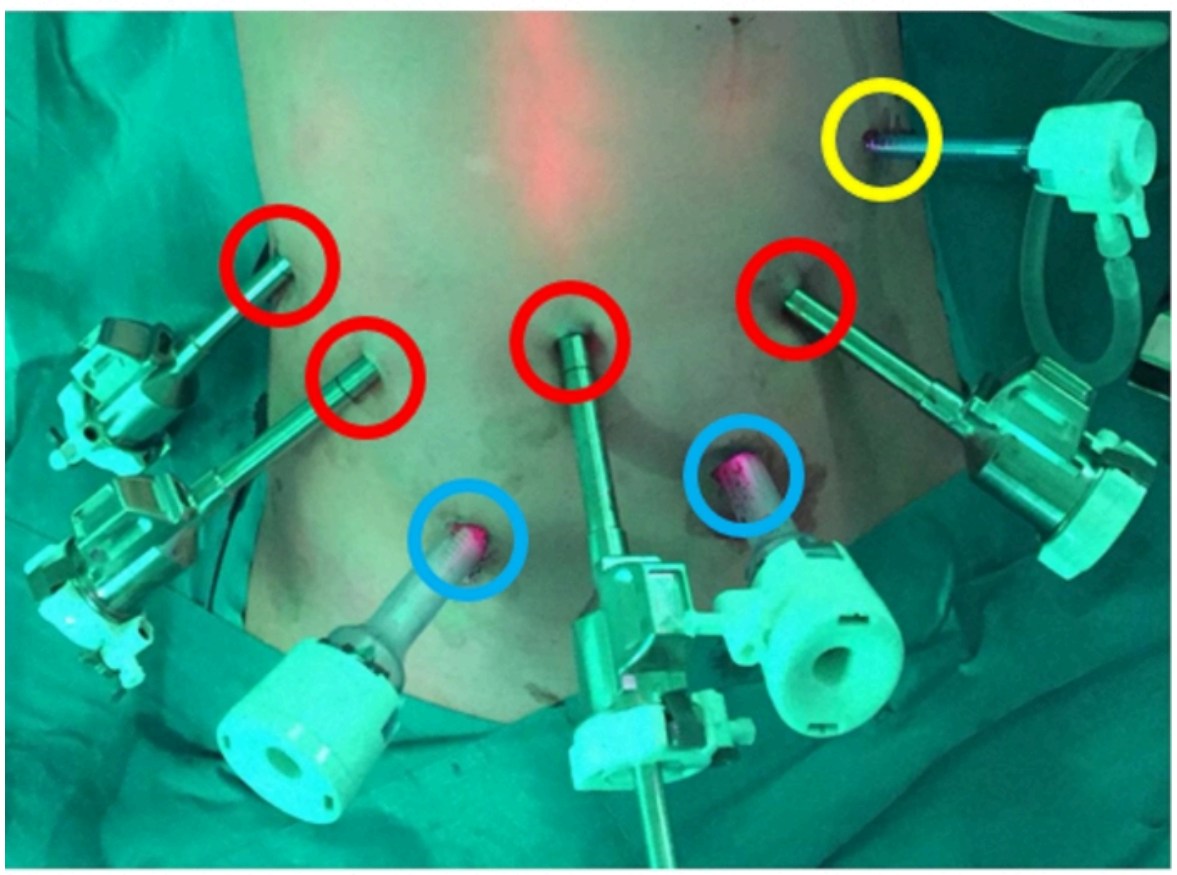

Figure 1: Trocar placement. Red: $8 \mathrm{~mm}$ robotic trocars, blue: $12 \mathrm{~mm}$ laparoscopic trocars, yellow: $5 \mathrm{~mm}$ trocar for stomach/ liver retractor. Please click here to view a larger version of this figure.

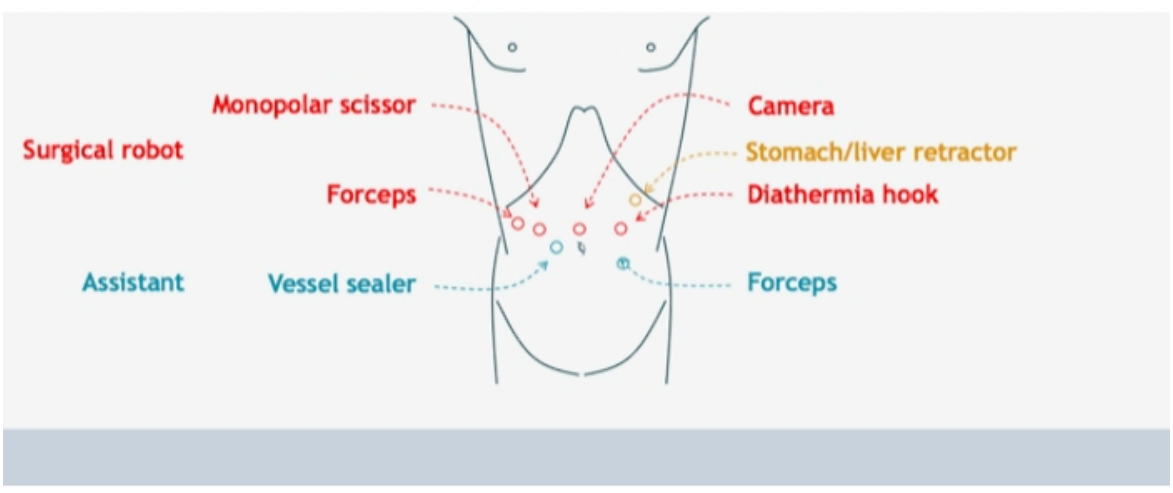

Figure 2: Schematic trocar placement. Please click here to view a larger version of this figure. 


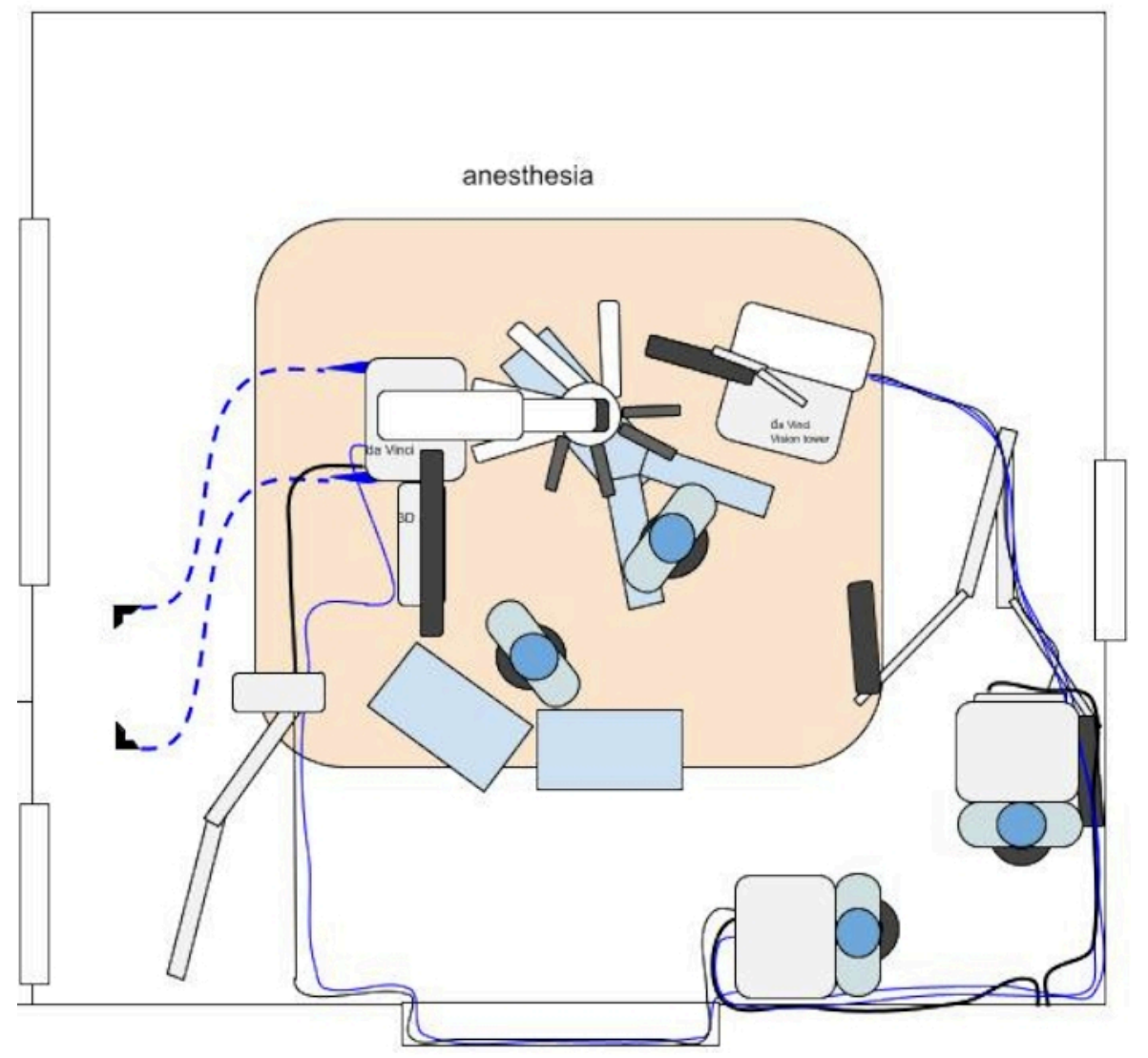

Figure 3: Set up and installation of the robot during surgery. Please click here to view a larger version of this figure. 


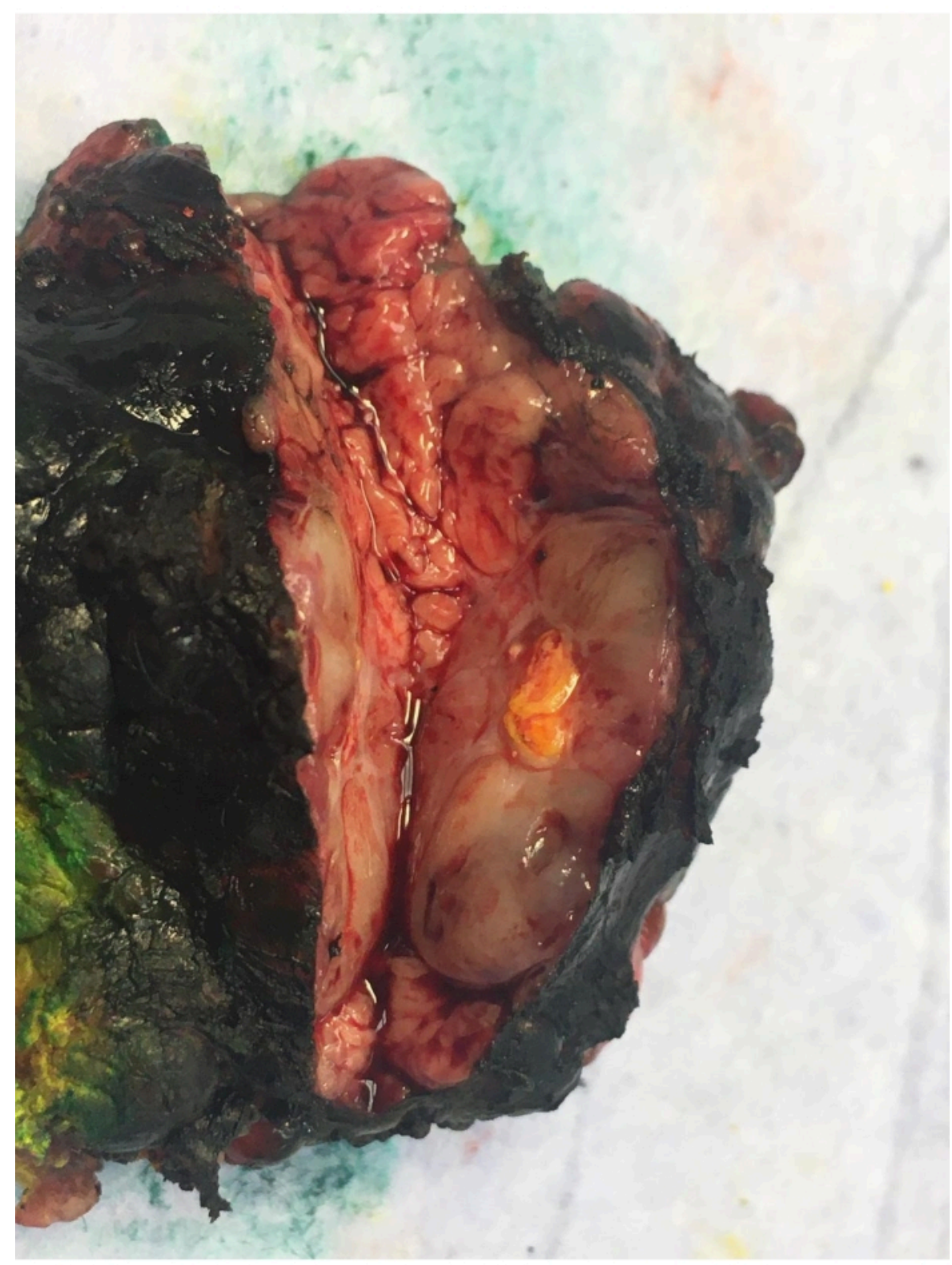

Figure 4: Macroscopic specimen. Please click here to view a larger version of this figure. 


\begin{tabular}{|c|c|}
\hline Variable & Outcome \\
\hline Intraoperative & 248 \\
\hline Operative time, minutes & 20 \\
\hline Intraoperative blood loss, $\mathrm{mL}$ & \\
\hline Postoperative & Grade B \\
\hline Postoperative pancreatic fistula (POPF) & 2 \\
\hline Clavien-Dindo complication grade & 7 \\
\hline Drain removal, postoperative day & 8 \\
\hline Postoperative hospital stay, days & Radically (R0) resected solid pseudopapillary \\
\hline Pathological diagnosis & tumor (i.e. Frantz tumor), 2.2 cm \\
\hline
\end{tabular}

Table 1: Representative results of the surgery.

\section{Discussion}

This report demonstrates that robotic central pancreatectomy with Roux-en-Y pancreaticojejunostomy is feasible in selected patients and in experienced hands. In our experience, the robotic approach has benefits compared to the laparoscopic approach, especially when an anastomosis has to be made, due to its enhanced technical capabilities such as surgeon controlled high definition three-dimensional vision, facilitated and magnified instrument movement, and inherently to this improved suturing control due to its wristed instruments ${ }^{18,19}$. In particular, our report demonstrated that the standard pancreatico-jejunal anastomosis from robotic pancreatoduodenectomy could be used.

A recent multicenter NSQIP analysis reported a reduced risk of POPF after robotic as compared to open pancreatoduodenectomy ${ }^{20}$. Therefore, the question arises whether robotic central pancreatectomy could reduce the risk of POPF when compared to open central pancreatectomy.

Nonetheless, patient characteristics and the risk of a POPF should be taken into account when determining whether to perform a central pancreatectomy. The rate of POPF after central pancreatectomy will remain higher than after distal pancreatectomy given the POPF rate of pancreaticojejunostomy. Therefore, central pancreatectomy should be reserved for patients who are at low risk of POPF or other complications. Whereas well-defined contra-indications for central pancreatectomy are lacking, not all patients with pre-malignant or low-grade malignant neoplasms in the body or neck of the pancreas are eligible. The most important selection criteria are probably the patient's age and condition and tumor characteristics as size and location. A central pancreatectomy would seem more indicated for a younger patient with a small tumor, good performance status, and 
no diabetes than an elderly patient already suffering from diabetes.

In addition to this, patient selection is as important as experience with the operative technique. It has to be noted that complication and mortality rates are reduced in centers performing at least 20 robotic pancreatoduodenectomy procedures per year. For this reason, the Miami guidelines advise to only perform this procedure in high volume centers by experienced surgeons ${ }^{18,21}$.

With regard to the choice of anastomosis, despite the good clinical outcomes of the pancreatico-jejunostomy in our report, the question remains how this anastomosis compares to a pancreatico-gastrostomy. No reliable evidence is available to support the use of one particular anastomosis ${ }^{22}$.

In conclusion, we have shown that robotic central pancreatectomy with Roux-en-Y pancreaticojejunostomy is a feasible and safe parenchyma-sparing minimally invasive alternative to open or laparoscopic central pancreatectomy or distal pancreatectomy. Unnecessary pancreatic resections with loss of parenchyma and thereby potential loss of longterm pancreatic function could thus be avoided. The general applicability in non-selected patients remains uncertain.

\section{Disclosures}

The authors have nothing to disclose.

\section{Acknowledgments}

We would like to acknowledge Melissa Hogg, Herbert Zeh III, Amer Zureikat, and Safi Dokmak for training and advice regarding robotic pancreatic surgery and central pancreatectomy. This clinical research was enabled by HPBAmsterdam.

\section{References}

1. Lacono, C. et al. Systematic review of central pancreatectomy and meta-analysis of central versus distal pancreatectomy. British Journal of Surgery. 100 (7), 873-885 (2013).

2. Xiao, W. et al. The role of central pancreatectomy in pancreatic surgery: a systematic review and metaanalysis. HPB (Oxford). 20 (10), 896-904 (2018).

3. Regmi, P. et al. Overall postoperative morbidity and pancreatic fistula are relatively higher after central pancreatectomy than distal pancreatic resection: $A$ systematic review and meta-analysis. Biomed Res Int. 2020, 7038907 (2020).

4. Dragomir, M. P., Sabo, A. A., Petrescu, G. E. D., Li, Y., Dumitrascu, T. Central pancreatectomy: a comprehensive, up-to-date meta-analysis. Langenbecks Archive of Surgery. 404 (8), 945-958 (2019).

5. Zhou, Y. M. et al. Pancreatic fistula after central pancreatectomy: case series and review of the literature. Hepatobiliary and Pancreatic Disease International. 13 (2), 203-208 (2014).

6. Goldstein, M. J., Toman, J., Chabot, J. A. Pancreaticogastrostomy: a novel application after central pancreatectomy. Journal of the American College of Surgeons. 198 (6), 871-876 (2004).

7. Sudo, T. et al. Middle pancreatectomy with pancreaticogastrostomy: A technique, operative outcomes, and long-term pancreatic function. Journal of Surgical Oncology. 101 (1), 61-65 (2010).

8. Borel, F. et al. Pancreatico-jejunostomy decreases postoperative pancreatic fistula incidence and severity after 
central pancreatectomy. ANZ Journal of Surgery. $\mathbf{8 8}$ (1-2), 77-81 (2018).

9. Qin, H., Luo, L., Zhu, Z., Huang, J. Pancreaticogastrostomy has advantages over pancreaticojejunostomy on pancreatic fistula after pancreaticoduodenectomy. A meta-analysis of randomized controlled trials. International Journal of Surgery. 36 (Pt A), 18-24 (2016).

10. Crippa, S. et al. Pancreaticojejunostomy is comparable to pancreaticogastrostomy after pancreaticoduodenectomy: an updated meta-analysis of randomized controlled trials. Langenbecks Archives of Surgery. 401 (4), 427-437 (2016).

11. Benini, L. et al. Residual pancreatic function after pancreaticoduodenectomy is better preserved with pancreaticojejunostomy than pancreaticogastrostomy: A long-term analysis. Pancreatology. 19 (4), 595-601 (2019).

12. Knab, L. M., Zureikat, A. H., Zeh, H. J., $3^{\text {rd }}$, Hogg, M. E. Towards standardized robotic surgery in gastrointestinal oncology. Langenbecks Archives of Surgery. 402 (7), 1003-1014 (2017).

13. Zwart, M. J. W. et al. Outcomes of a multicenter training program in robotic pancreatoduodenectomy (LAELAPS-3). Annals of Surgery. (2021).

14. Jones, L. R. et al. Robotic pancreatoduodenectomy: patient selection, volume criteria, and training programs. Scandinavian Journal of Surgery. 109 (1), 29-33 (2020).

15. Asbun, H. J. et al. Technique and audited outcomes of laparoscopic distal pancreatectomy combining the clockwise approach, progressive stepwise compression technique, and staple line reinforcement. Surgical Endoscopy. 34 (1), 231-239 (2020).
16. Hogg, M. E. et al. Training in minimally invasive pancreatic resections: a paradigm shift away from "see one, do one, teach one". HPB (Oxford). 19 (3), 234-245 (2017).

17. Bassi, C. et al. The 2016 update of the International Study Group (ISGPS) definition and grading of postoperative pancreatic fistula: 11 years after. Surgery. 161 (3), 584-591 (2017).

18. Wright, G. P., Zureikat, A. H. Development of minimally invasive pancreatic surgery: an evidencebased systematic review of laparoscopic versus robotic approaches. Journal of Gastrointestinal Surgery. 20 (9), 1658-1665 (2016).

19. Eid, G. M., Entabi, F., Watson, A. R., Zuckerbraun, B. S., Wilson, M. A. Robotic-assisted laparoscopic side-to-side lateral pancreaticojejunostomy. Journal of Gastrointestinal Surgery. 15 (7), 1243 (2011).

20. Vining, C. C. et al. Robotic pancreaticoduodenectomy decreases the risk of clinically relevant post-operative pancreatic fistula: a propensity score matched NSQIP analysis. HPB (Oxford). 23 (3), 367-378 (2021).

21. Asbun, H. J. et al. The Miami International Evidencebased Guidelines on Minimally Invasive Pancreas Resection. Annals of Surgery. 271 (1), 1-14 (2020).

22. Lyu, Y. et al. Pancreaticojejunostomy Versus Pancreaticogastrostomy After Pancreaticoduodenectomy: An Up-to-date metaanalysis of RCTs applying the ISGPS (2016) Criteria. Surgical Laparoscopy Endoscopy and Percutaneous Techiques. 28 (3), 139-146 (2018). 